\title{
Violência sexual e coocorrências em crianças e adolescentes: estudo das incidências ao logo de uma década
}

\author{
Sexual violence and co-occurrences suffered by children \\ and adolescents: study of incidents over a decade
}

Jacqueline Reiter de Oliveira ${ }^{1}$

Maria Conceição Oliveira Costa ${ }^{1}$

Magali Teresópolis Reis Amaral ${ }^{1}$

Clarice Alves Santos ${ }^{1}$

Simone Gonçalves de Assis ${ }^{2}$

Ohana Cunha do Nascimento ${ }^{1}$

\footnotetext{
${ }^{1}$ Programa de PósGraduação em Saúde Coletiva, Departamento de Saúde, Universidade Estadual de Feira de Santana. Av. Transnordestina S/N Campus Universitário - Módulo VI, Novo Horizonte. 44.031-460 Feira de Santana BA Brasil. jac.reiter@gmail.com ${ }^{2}$ Centro Latino-Americano de Estudos de Violência e Saúde Jorge Carelli, Escola Nacional de Saúde Pública Sergio Arouca, Fundação Osvaldo Cruz.
}

\begin{abstract}
The study analyzes the evolution of the incidence of sexual violence (SV) and co-occurrences between 2001 and 2010. The records of the Guardianship Councils in Feira de Santana, State of Bahia, Brazil were used and the incidence rates and graphs of the events during the period were calculated. Of the total of the different types of violence, $21.8 \%$ involved co-occurrences, the majority being female, most frequently during adolescence. There was a high proportion of abuse in male children, with most offenders bring family members or acquaintances. The incidence of $S V$ revealed an increasing trend in both sexes during the decade, more significantly in females in 2002 and 2009. The age groups indicated the same trend, with a higher proportion of cases in adolescence. The record of co-occurrences with $S V$ was more pronounced in the second half of the decade, namely psychological violence in 2008, neglect in 2008 and physical violence in 2009. The conclusion is that the increase in the coefficients of sexual violence and co-occurrences may indicate an improvement of the reporting system of instances in reference, as well as greater citizen participation through the Dial 100 complaint hotline. The indicators help to prevent and control violence against children.
\end{abstract}

Key words Child and adolescent, Sexual violence, Maltreatment, Exposure to violence, Incidence
Resumo O presente estudo analisa a evolução da incidência da violência sexual (VS) e coocorrências, ao longo de 2001 a 2010. Constitui série histórica de casos a partir dos Conselhos Tutelares e CREAS de Feira de Santana (BA), Brasil. Foram calculados coeficientes de incidência da violência sexual e outras violências concomitantes e elaboradas curvas desses eventos. No total dos casos de violência sexual registrados, 21,8\% tiveram outras coocorrências; a maioria do sexo feminino, com maior frequência na adolescência, embora com alta proporção de abuso em crianças do sexo masculino; a maior parte dos agressores era conhecido ou familiar. A incidência da VS mostrou tendência crescente em ambos os sexos, ao longo da década, expressivamente no feminino: em 2002 e 2009; as faixas etárias mostraram a mesma tendência, com maior proporção de casos na adolescência. O registro das coocorrências à VS foi mais expressivo na $2^{a}$ metade da década: violência psicológica em 2008; negligência em 2008; violência física, em 2009. O aumento dos coeficientes de VS e coocorrências sugerem aprimoramento do sistema de notificação das instâncias de referência, bem como maior participação popular, através do Disque 100. Os indicadores contribuem para a prevenção e controle da violência infanto-juvenil.

Palavras-chave Criança e adolescente, Violência sexual, Maus tratos, Exposição à violência, Incidência 


\section{Introdução}

Nos diferentes contextos socioculturais e países, a violência tem sido apontada como a principal causa de morbimortalidade na região das Américas, países desenvolvidos e em desenvolvimento ${ }^{1,2}$, provocando mobilização mundial de instâncias internacionais voltadas à defesa dos direitos humanos ${ }^{3}$, na busca de mecanismos eficientes para investigar os casos, cumprir as leis e responsabilizar os envolvidos ${ }^{4}$.

A violência sexual de crianças e adolescentes, além de um grave problema de saúde pública, constitui um crime violento ${ }^{5}$, reconhecido como um desafio social ${ }^{6}$, em decorrência das consequências físicas, emocionais, como estresse pós-traumático ${ }^{7}$, entre outros comprometimentos ligados ao comportamento e desenvolvimento cognitivo e psicossocial ${ }^{5,8,9}$. Em nível mundial, estudos apontam que, independente do contexto social e econômico, meninas e meninos, antes mesmo de alcançar a maioridade, podem sofrer violência sexual, cujos índices divergem, em virtude de múltiplos fatores ${ }^{5,6,8}$, como aspectos ligados à dinâmica da revelação, pela vítima e dificuldades na efetivação da denúncia e investigação do caso, além de fatores culturais e preconceitos ${ }^{10}$.

Embora a maioria dos estudos nesta área enfoque apenas um tipo de vitimização ${ }^{11-14}$, pesquisadores afirmam que é frequente a presença de outros episódios violentos, concomitantes à ocorrência da violação sexual, onde os baixos coeficientes mostrados nas estatísticas oficiais contribuem com a impunidade e não retrata a real magnitude do fenômeno ${ }^{8,13,15-17}$. Estudos que investigam formas individuais de violência são susceptíveis de subestimar outras experiências violentas?. Entre os fatores que predispõem à ocorrência de múltiplas violências destacam-se a inserção em comunidades com graves problemas sociais, família exposta a situações de perigo e o convívio em ambiente familiar caótico e problemático ${ }^{18,19}$.

A identificação de crianças e adolescentes expostos aos diversos tipos de vitimização concomitantes possibilita conclusões mais precisas sobre o problema, conhecimento da real magnitude do fenômeno, bem como o efeito cumulativo das adversidades. Vale ressaltar que as vítimas podem apresentar alto nível de sofrimento psicológico, risco de comprometimento psiquiátrico e vulnerabilidade presumida para outros agra$\operatorname{vos}^{20}$. Problemas de origem emocional são apontados na literatura como preditores da exposição e, consequentemente, podem levar à delinquência juvenil ${ }^{21}$.
A elaboração, o monitoramento e a avaliação dos indicadores de violência perpetrada sobre crianças e adolescentes são imprescindíveis à difusão do conhecimento sobre as tendências do fenômeno, nas diferentes populações, assim como subsidiam o planejamento e a implementação de políticas e programas estratégicos de prevenção e intervenção $0^{4}$. No Brasil, esforços têm sido empreendidos, pelos diversos setores, na operacionalização do Sistema Nacional de Denúncia Anônima "Disque 100"22 e do Sistema de Vigilância de Acidentes e Violências - VIVA/MS. Avanços na produção e divulgação do conhecimento nesta área resultam de parcerias e intercâmbios interinstitucionais entre Universidades, Centro de Pesquisas e Sistemas de Atendimento e Defesa de Direitos ${ }^{17}$.

No que diz respeito à participação de Feira de Santana na Rede de Prevenção e Atendimento às vítimas de violência, vale ressaltar que o município integra o Programa Nacional de Enfrentamento da Violência Infanto-Juvenil, desde 2003, compondo o grupo de cidades consideradas de alto risco para violação, tráfico e exploração de crianças e adolescentes, pelas características econômicas e geográficas (polo econômico e industrial do Estado, portal da região do semiárido da Bahia e situada em um dos importantes trevos rodoviários do país, ligação entre as regiões norte, nordeste e sudeste do país (através de rodovias federais e estaduais), com alto fluxo migratório das regiões mais pobres, em direção às maiores cidades e a capital Salvador, importante polo turístico do país. Em 2003, Feira de Santana assinou o pacto de participação e implantação da Rede de Atendimento, Garantia e Defesa de Direitos de crianças e adolescentes, integrando instâncias de diferenciados setores com a participação da Universidade. Integrante desta Rede, a Universidade Estadual de Feira de Santana (UEFS) assume papel integrador, contribuindo no fortalecimento das instituições, na formação de recursos humanos, no levantamento de indicadores, na formação de novos pesquisadores e subsidiando o poder público na implementação das estratégias e intervenções direcionadas ao enfrentamento dessa demanda social ${ }^{14,15}$. Este processo vem sendo incrementado através da articulação interinstitucional entre os níveis federal e municipal, com apoio da Organização Internacional do Trabalho (OIT), Fundação de Amparo à Pesquisa da Bahia (FAPESB), entre outras instâncias de fomento à pesquisa $(\mathrm{CNPq}, \mathrm{MEC})^{23}$.

O presente artigo tem como objetivo analisar os coeficientes de incidência da violência sexual 


\section{Método}

Estudo epidemiológico do tipo série de casos, com base em dados secundários, obtidos a partir dos registros de violência sexual e coocorrências existentes nos dois Conselhos Tutelares I e II e CREAS existentes em Feira de Santana, Bahia/Brasil.

Com a finalidade de analisar a evolução do fenômeno, optou-se por estudar o período de 2001 a 2010, considerando a adesão de Feira de Santana às ações e metas do "Programa Nacional de Ações Direcionadas ao Enfrentamento da Violência Sexual/PAIR", momento em que foram implementadas (2003) e avaliadas (2009) as ações integradas entre a "Rede" de instâncias de atendimento e de defesa (Saúde, Educação e Assistência, Conselhos Tutelares, CREAS, outros). Os projetos desenvolvidos consolidaram parcerias entre a Rede de Instâncias e a Universidade Estadual de Feira de Santana com o Núcleo de Estudos e Pesquisa na Infância e Adolescência UEFS/NNEPA.

A população de estudo compreende crianças ( $\leq 11$ anos) e adolescentes (12-18 anos), de ambos os $\operatorname{sexos}^{24}$, registradas nas instâncias de referência (CT e CREAS), vítimas de violência sexual e outras concomitantes, cujos dados foram coletados dos prontuários de atendimento registrados no período. Foi considerada como coocorrência a presença de um ou mais tipo de violência concomitante à sexual ${ }^{25-27}$. O formulário de coleta foi testado por estudos anteriores do NNEPA/ $\mathrm{UEFS}^{23}$.

Os dados foram processados eletronicamente no Statistical Package for the Sciences (SPSS), versão 9.0 for Windows. Inicialmente, realizou-se o linkage entre os bancos de dados dos Conselhos Tutelares e CREAS, tendo em vista evitar duplicidade de registro ${ }^{28}$. Em seguida foram calculadas as medidas de frequências simples e relativas das variáveis e calculados os coeficientes de incidência da violência sexual e das outras ocorridas no mesmo evento, segundo sexo e faixa etária das vítimas e ano (2001-2010). Como base para o cálculo, foi utilizada a população estimada para a mesma faixa etária e ano, residentes em Feira de Santana, no período, para cada 100.000 crianças ou adolescentes em acordo aos dados do Instituto Brasileiro de Geografia e Estatística (IBGE).

\section{Resultados}

São analisadas a seguir 1.418 notificações de violência sexual perpetradas em crianças e adolescentes no período de 2001 a 2010, com média de notificação anual de 142 registros, cerca de 10 casos ao mês e três a cada semana.

$\mathrm{Na}$ Tabela 1 verifica-se que a maior proporção dos casos de violência sexual foi registrado nos Conselhos Tutelares (cerca de 58,2\%), sendo a maioria sem outros eventos violentos concomitantes $(78,2 \%)$. Independente da presença das coocorrências, a violência sexual se manifestou como estupro, incesto e exploração e violência psicológica, quando analisada em concomitância com outros tipos de eventos violentos, permaneceu como pano de fundo em $10,9 \%$ dos casos. A maioria dos casos registrados ocorreu no domicílio da vítima e em outros espaços da comunidade; a principal forma de denúncia foi através do Sistema "Disque 100" ou pela mãe da vítima; os encaminhamentos mais encontrados foram para os Conselhos Tutelares, CREAS e Delegacias especializadas.

$\mathrm{Na}$ Tabela 2, os resultados mostram que a maioria das vítimas era do sexo feminino, com maior número de casos na adolescência, entretanto, com alta proporção de vitimização nas faixas da infância. Em relação ao agressor, independentemente da existência das coocorrências, verifica-se que se tratava de pessoa conhecida da vítima ou membro da família, incluindo pai e mãe, a maior parte do sexo masculino, valendo assinalar que, embora os dados dos perpetradores tenham apresentado baixa captação (ausên- 
Tabela 1. Características do vitimização sexual contra crianças e adolescentes, segundo dados da violência sexual isolada e em coocorrência com outras violências. Conselhos Tutelares e CREAS, 2001 a 2010. Feira de Santana, BA, Brasil.

\begin{tabular}{|c|c|c|c|c|}
\hline \multirow{2}{*}{ Características da vitimização } & \multicolumn{2}{|c|}{ Violência sexual isolada } & \multicolumn{2}{|c|}{ Violência sexual com coocorrência } \\
\hline & $\mathbf{n}$ & $\%$ & $\mathbf{n}$ & $\%$ \\
\hline \multicolumn{5}{|l|}{ Instituições de atendimento } \\
\hline CT I e II & 593 & 41,8 & 232 & 16,4 \\
\hline CREAS & 517 & 36,4 & 76 & 5,4 \\
\hline Total & 1110 & 78,2 & 308 & 21,8 \\
\hline \multicolumn{5}{|l|}{ Tipos de violência sexual } \\
\hline Estupro & 404 & 28,8 & 84 & 6,0 \\
\hline Incesto & 340 & 24,3 & 135 & 9,6 \\
\hline Exploração/Prostituição & 248 & 17,7 & 55 & 3,9 \\
\hline Outros tipos & 90 & 6,5 & 45 & 3,2 \\
\hline Total & 1082 & 77,3 & 319 & 22,7 \\
\hline \multicolumn{5}{|l|}{ Coocorrência de outras violências ${ }^{*}$} \\
\hline Violência física e outras & - & - & 120 & 8,5 \\
\hline Violência psicológica e outras & - & - & 155 & 10,9 \\
\hline Negligência e outras & - & - & 119 & 8,4 \\
\hline Total & - & - & 394 & 27,8 \\
\hline \multicolumn{5}{|l|}{ Local de ocorrência } \\
\hline Casa da vítima & 485 & 39,4 & 210 & 17,1 \\
\hline Outros espaços da comunidade ${ }^{b}$ & 466 & 37,9 & 69 & 5,6 \\
\hline Total & 951 & 77,3 & 279 & 22,7 \\
\hline \multicolumn{5}{|l|}{ Denunciante } \\
\hline Sistema "Disque 100" (anônimo) & 225 & 17,4 & 84 & 6,5 \\
\hline Mãe & 255 & 19,7 & 27 & 2,1 \\
\hline Conselho Tutelar & 87 & 6,7 & 12 & 0,9 \\
\hline Outros denunciantes ${ }^{\mathrm{c}}$ & 450 & 34,8 & 152 & 11,8 \\
\hline Total & 1017 & 78,7 & 275 & 21,3 \\
\hline \multicolumn{5}{|l|}{ Encaminhamentos Institucionais ${ }^{*}$} \\
\hline Conselho Tutelar & 566 & 33,6 & 179 & 10,6 \\
\hline Sentinela/CREAS & 430 & 25,5 & 90 & 5,4 \\
\hline Delegacias & 183 & 10,9 & 59 & 3,5 \\
\hline Serviços de Saúde & 48 & 2,8 & 11 & 0,6 \\
\hline Outros $^{\mathrm{d}}$ & 82 & 4,9 & 37 & 2,2 \\
\hline Total & 1309 & 77,7 & 376 & 22,3 \\
\hline
\end{tabular}

a Outros tipos: sedução $(\mathrm{n}=56)$; pornografia $(\mathrm{n}=40)$; assédio sexual $(\mathrm{n}=3)$; aliciamento $(\mathrm{n}=6)$; exibicionismo $(\mathrm{n}=21)$; Voyeurismo $(n=7)$; Tráfico $(n=2){ }^{\text {b }}$ Outros espaços da comunidade: casa do agressor $(n=105)$; outros espaços $(n=430)$. c Outros denunciantes: outros familiares $(\mathrm{n}=122)$, Sistema de Garantias de Direitos (Delegacias e Policias; Ministério Público; Defensoria Pública; Juizado Infanto-Juvenil e outros $) \mathrm{n}=249$; Educação $(\mathrm{n}=56)$; Saúde $(\mathrm{n}=50)$; Assistência $(\mathrm{n}=64)$. ${ }^{\mathrm{d}}$ Outros: Juizado $(n=61)$; Ministério Público e Defensoria Pública $(n=39)$. ${ }^{*}$ Múltipla resposta: um caso pode sofrer mais de um tipo de violência.

cia de registro de algumas variáveis), observouse alta proporção da intimidação pela força física e ameaças, com alta frequência de agressor adolescente e adulto jovem.

Na Figura 1 em que são apresentados os coeficientes de incidência da violência sexual isolada e com coocorrência de outras violências, verificouse que, independentemente da existência de coocorrência, houve aumento significativo do registro de casos, ao longo da década: 2002 (77,1 casos por cem mil habitantes - um menino para cada
6,5 meninas); 2008 (120,5 casos - um menino para cada sete meninas) e 2009 (122 casos - um menino para cada 6,7 meninas). Os coeficientes da violência sexual que ocorre isoladamente apresentam perfil similar, com taxas mais elevadas nos anos de 2007, 2008 e 2009. Na presença das coocorrências, os maiores coeficientes da violência sexual foram observados ao final do período: em 2008 (36,5 casos por cem mil habitantes - um menino para cada cinco meninas) e $2009(37,1$ casos - um menino para cada 4,3 meninas). 
Tabela 2. Perfil das crianças e adolescentes vítimas e dos agressores, segundo violência sexual isolada e em coocorrência com outras violências. Conselhos Tutelares e CREAS, 2001 a 2010. Feira de Santana, BA, Brasil.

\begin{tabular}{|c|c|c|c|c|}
\hline \multirow{2}{*}{$\begin{array}{c}\text { Características da vítima } \\
\text { e do agressor }\end{array}$} & \multicolumn{2}{|c|}{ Violência sexual isolada } & \multicolumn{2}{|c|}{ Violência sexual com coocorrência } \\
\hline & $\mathbf{n}$ & $\%$ & n & $\%$ \\
\hline \multicolumn{5}{|l|}{ Sexo da vítima (1230) } \\
\hline Masculino & 178 & 12,6 & 62 & 4,4 \\
\hline Feminino & 932 & 65,7 & 246 & 17,3 \\
\hline Total & 1110 & 78,3 & 308 & 21,7 \\
\hline \multicolumn{5}{|l|}{ Faixa etária da vítima (1314) } \\
\hline Infância até 11 anos & 410 & 31,2 & 130 & 9,9 \\
\hline Adolescência 12 a 18 & 613 & 46,7 & 161 & 12,3 \\
\hline Total & 1023 & 77,9 & 291 & 22,1 \\
\hline \multicolumn{5}{|l|}{ Responsável pela vítima* } \\
\hline Pai & 198 & 15,2 & 57 & 4,4 \\
\hline Mãe & 764 & 58,7 & 188 & 14,5 \\
\hline Outros $^{\mathrm{a}}$ & 66 & 5,1 & 28 & 2,1 \\
\hline Total & 1028 & 79,0 & 273 & 21,0 \\
\hline \multicolumn{5}{|l|}{ Vínculo do agressor com a vítima* } \\
\hline Pai ou mãe & 156 & 10,7 & 97 & 6,7 \\
\hline Padrasto ou madrasta & 100 & 6,9 & 48 & 3,3 \\
\hline Outros familiares ${ }^{\mathrm{b}}$ & 93 & 6,4 & 30 & 2,1 \\
\hline Conhecidos da família ou da vítima ${ }^{\mathrm{c}}$ & 597 & 40,9 & 110 & 7,5 \\
\hline Desconhecidos & 193 & 13,2 & 34 & 2,3 \\
\hline Total & 1139 & 78,1 & 319 & 21,9 \\
\hline \multicolumn{5}{|l|}{ Sexo do agressor (1251) } \\
\hline Masculino & 797 & 63,7 & 228 & 18,2 \\
\hline Feminino & 160 & 12,8 & 66 & 5,3 \\
\hline Total & 957 & 76,5 & 294 & 23,5 \\
\hline \multicolumn{5}{|l|}{ Faixa etária do agressor (532) } \\
\hline Até 17 anos & 143 & 26,9 & 38 & 7,1 \\
\hline $19-39$ & 175 & 32,9 & 53 & 10,0 \\
\hline 40 e mais & 92 & 17,3 & 31 & 5,8 \\
\hline Total & 410 & 77,1 & 122 & 22,9 \\
\hline \multicolumn{5}{|l|}{ Uso intimidação (828) } \\
\hline $\operatorname{Sim}$ & 451 & 54,5 & 184 & 22,2 \\
\hline Não & 164 & 19,8 & 29 & 3,5 \\
\hline Total & 615 & 74,3 & 213 & 25,7 \\
\hline \multicolumn{5}{|l|}{ Tipos de intimidação* } \\
\hline Força física & 177 & 25,2 & 123 & 17,5 \\
\hline Ameaça & 283 & 40,3 & 111 & 15,8 \\
\hline Arma fogo/ou faca & 7 & 1,0 & 2 & 0,3 \\
\hline Total & 467 & 66,5 & 236 & 33,6 \\
\hline
\end{tabular}

* Múltiplas respostas. ${ }^{\mathrm{a}}$ Outros responsáveis pelas vítimas: avós $(\mathrm{n}=61)$; tios $(\mathrm{n}=33)$. ${ }^{\mathrm{b}}$ Agressor - Outros familiares: irmãos $(\mathrm{n}=38)$; tios $(\mathrm{n}=61)$; avós $(\mathrm{n}=24) .{ }^{\mathrm{c}}$ Conhecidos da família: vizinhos $(\mathrm{n}=179)$; outros conhecidos $(\mathrm{n}=528)$.

As outras violências concomitantes à violência sexual mostraram considerável aumento da incidência, na segunda metade da década: violência psicológica, 9,0 casos em cem mil habitantes em 2005 e 10,5 casos em 2008; negligência, 10,0 casos em 2008 e 9,5 casos em 2009; violência física, 6,5 casos em 2008 e 8,0 casos em 2009.
Na Figura 2, a distribuição dos dados segundo o sexo mostra altos coeficientes da violência sexual isolada no sexo feminino, tanto no início da década, 2002 (119,3 casos em cem mil habitantes), quanto ao final da mesma, 2008 (147,2 casos) e 2009 (148,7 casos). Enquanto que, no sexo masculino, essas taxas foram mais expres- 
Violência sexual geral, isolada e em coocorrência

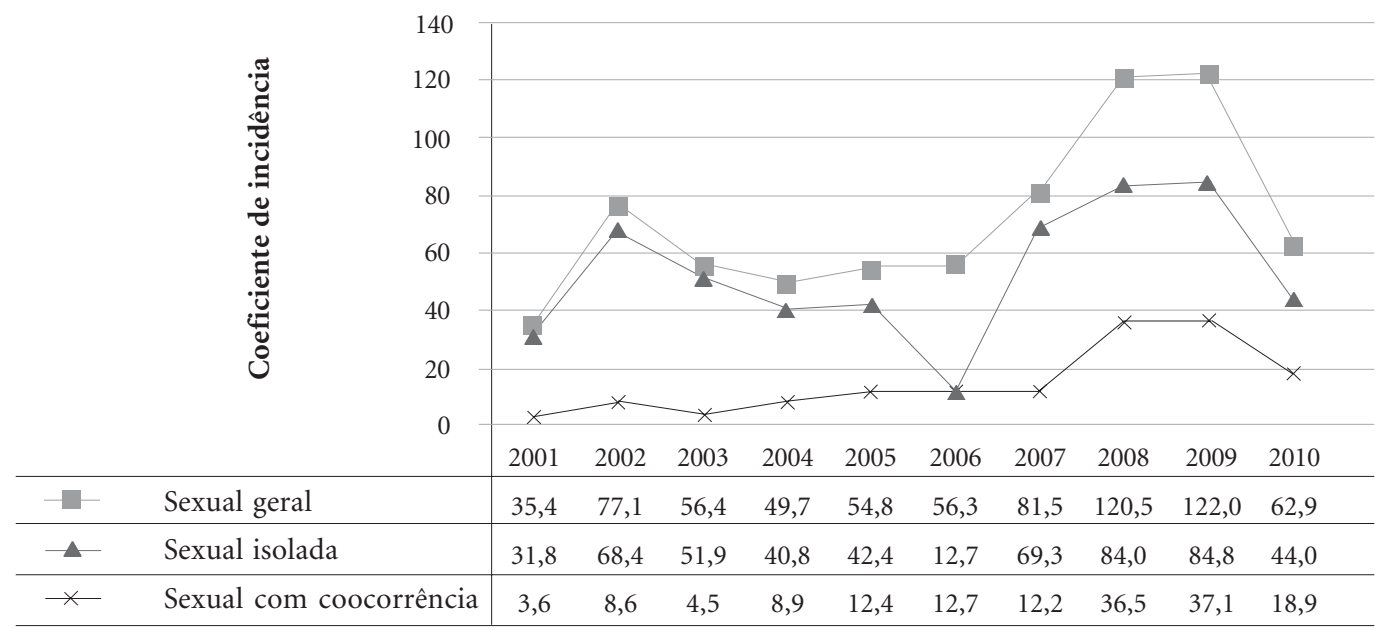

Coocorrência de outras violências à violência sexual

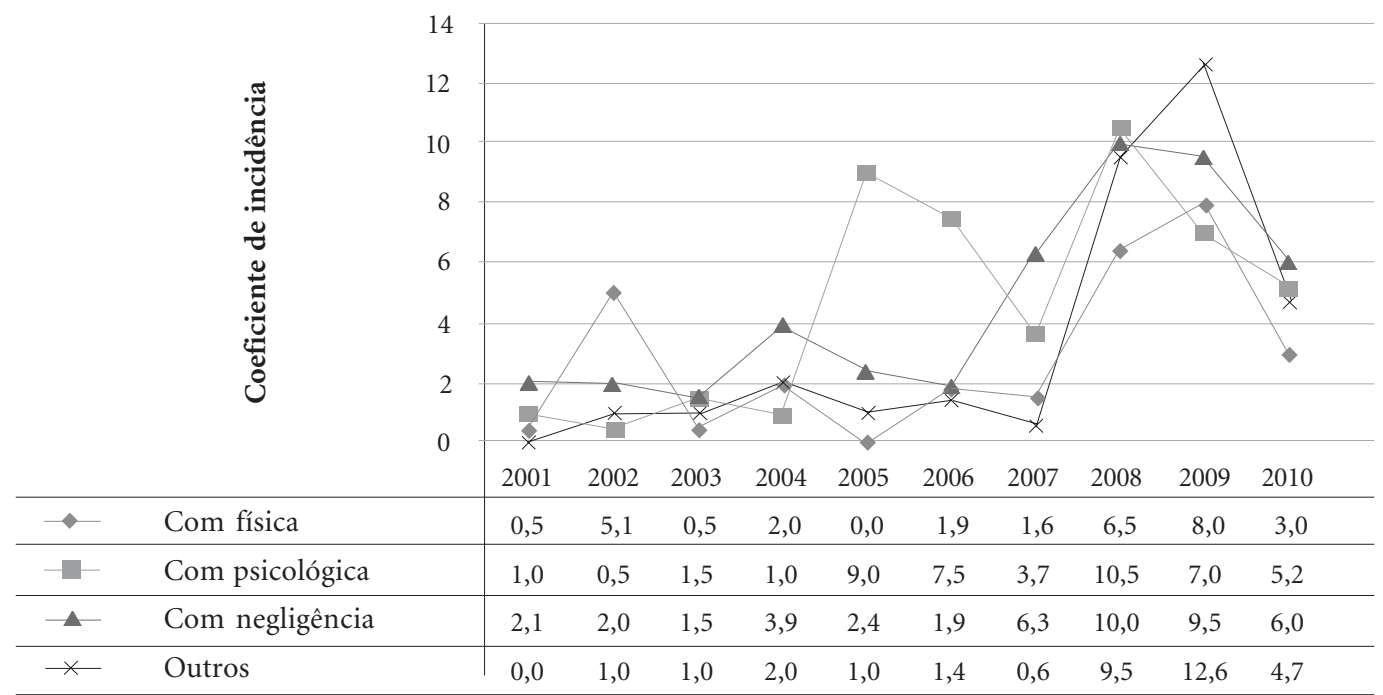

Figura 1. Coeficientes de incidência (por 100 mil habitantes) da violência sexual (isolada e com coocorrências), em crianças e adolescentes. Conselhos Tutelares e CREAS de Feira de Santana, BA, 2001 a 2010.

sivas ao final do período, 2008 (20,8 casos) e 2009 (21,9 casos). Dentre os casos com coocorrência, verifica-se tendência de maiores coeficientes ao fim da década, para ambos os sexos.

$\mathrm{Na}$ distribuição por faixa etária (Figura 3) destaca-se a fase da adolescência. Foi observado que, especialmente ao final do período estudado, foram verificados maiores coeficientes de violência sexual entre adolescentes, independente da presença das coocorrências. Este perfil também foi observado entre crianças, porém com taxas menos expressivas.

\section{Discussão}

Ao iniciar a discussão dos indicadores de violência sexual ao longo de uma década, vale assinalar 
Violência sexual isolada

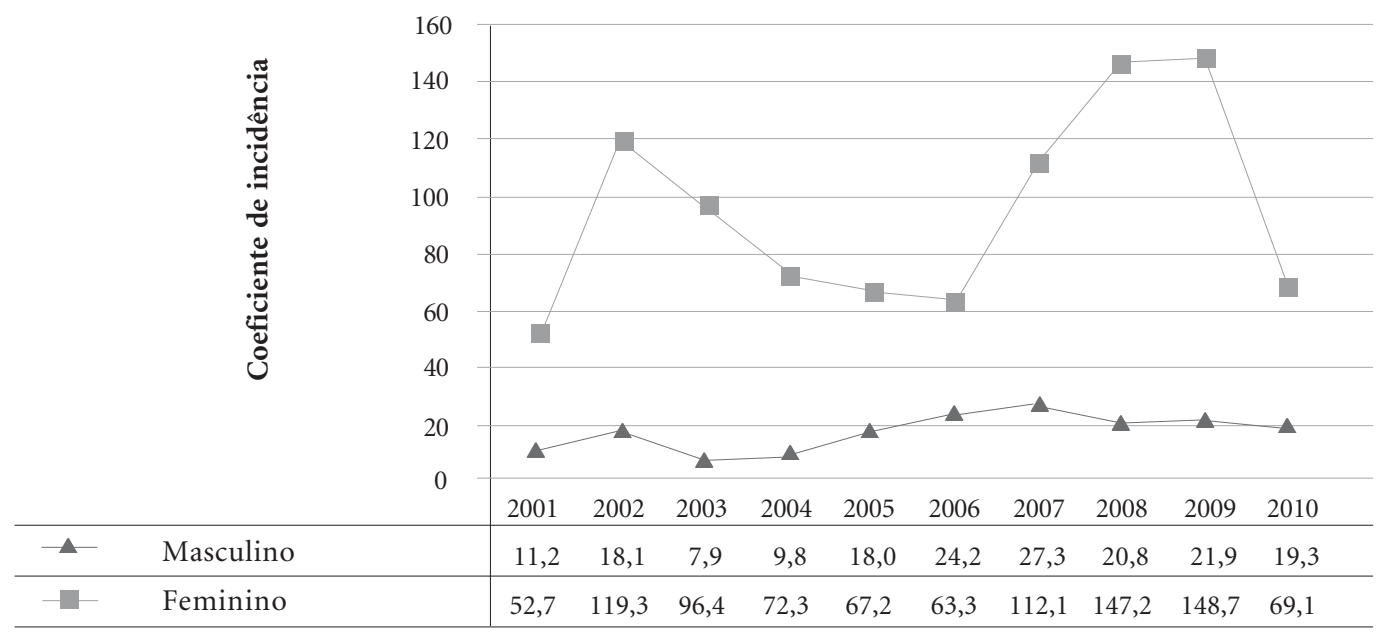

Coocorrência de outras violências à violência sexual

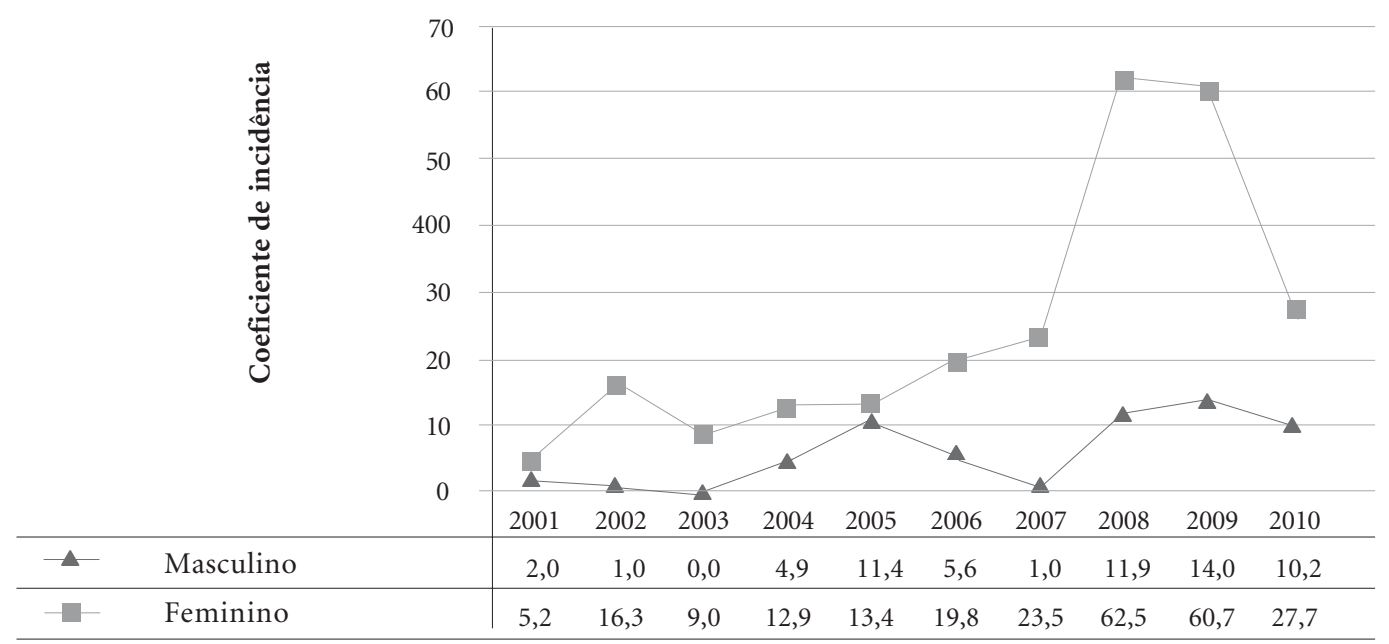

Figura 2. Coeficientes de incidência (por 100 mil habitantes) da violência sexual (isolada e com coocorrências), em crianças e adolescentes, segundo sexo das vítimas. Conselhos Tutelares e CREAS de Feira de Santana, BA, 2001 a 2010.

o consenso dos estudos na área que apontam inúmeras dificuldades para revelação e notificação dos casos desse tipo de violação. Pesquisas realizadas em distintos contextos e países mostram limitações técnicas e operacionais para a viabilização de dados, independente da fonte de informação (primária ou secundária), metodologia utilizada (qualitativa e/ou quantitativa) e área de concentração estudada (saúde, educação, assistência social, psicologia, direito, outras). Es- ses aspectos costumam estar ligados ao tempo de exposição da vítima, vínculo com o agressor, local de ocorrência, período etário da vítima, entre outros obstáculos que impedem ou retardam a revelação, pela vítima, ou a denúncia pela família ou terceiros ${ }^{30-33}$.

Os resultados do presente estudo, que mostraram aumento dos coeficientes de incidência da violência sexual ao longo de dez anos, concordam com pesquisas realizadas em Conselhos 
Violência sexual isolada

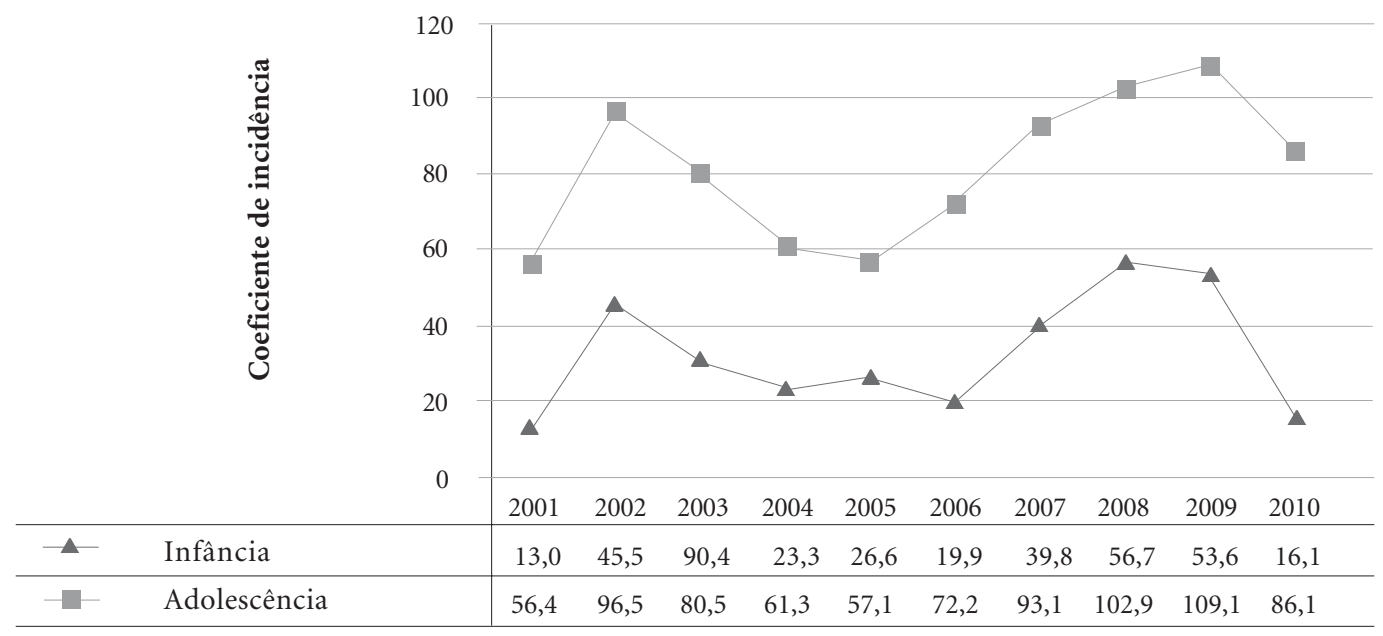

Coocorrência de outras violências à violência sexual

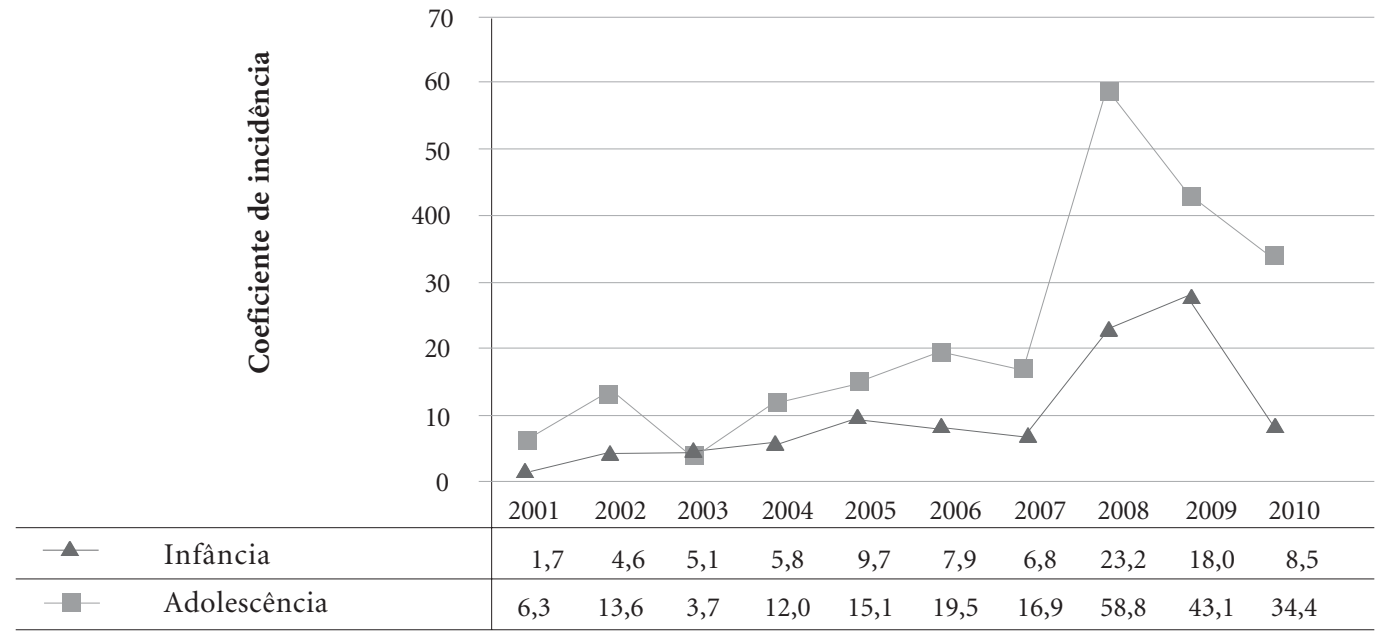

Figura 3. Coeficientes de incidência (por 100 mil habitantes) da violência sexual (isolada e com coocorrências), em crianças e adolescentes, segundo faixa etária das vítimas. Conselhos Tutelares e CREAS de Feira de Santana, BA, 2001 a 2010.

Tutelares de outros municípios do Brasil, no mesmo período, os quais também verificaram resultados semelhantes (por exemplo, 306 casos/ 100.000, Londrina, PR, em 2006) $)^{34}$. A violência sexual, independente da presença de outras ocorrências violentas concomitantes, merece atenção, pela gravidade e comprometimento à saúde física e psicossocial das vítimas, assim como pela invisibilidade decorrente da subnotificação envolvida no processo de vitimização ${ }^{14,27,34}$.
O aumento expressivo das incidências de violência sexual isolada e em coocorrência com outras, evidenciado no presente estudo a partir da segunda metade do decênio, pode estar relacionado às melhorias na capacidade de registro dos Conselhos Tutelares e à atuação em "Rede" das demais instâncias de atenção, face à identificação e encaminhamentos dos casos, assim como a atuação da comunidade, através das denúncias pelo Sistema Nacional - "Disque 100". Estes resulta- 
dos sinalizam mudanças positivas, quanto ao processo de sensibilização popular e participação das instâncias, ao mesmo tempo em que instiga a necessidade de multiplicar esforços, no sentido de fortalecimento institucional, na qualidade do atendimento, diante dos casos, assim como, na qualidade do sistema de registro de dados ${ }^{31}$.

Corroborando com achados de pesquisas realizadas e distintos contextos e países, os resultados do estudo de Feira de Santana mostraram que a violência sexual acometeu, especialmente, o sexo feminino, na maioria dos casos na adolescência (12-15 anos $)^{10}$, onde interferem fatores culturais que, historicamente, impuseram às mulheres condições de abuso e discriminação, socialmente aceitas ${ }^{10,14,31-34}$. Entretanto, vale ressaltar as altas proporções de vitimização na infância (até 11 anos), no sexo masculino, entre os resultados do presente estudo, especialmente, a partir de seis anos, concordando com outras pesquisas que verificaram alta frequência de violação no sexo masculino, nas faixas da infância. Esses achados, possivelmente, estão relacionados a fatores culturais, que cursam com estigmatização, preconceitos e outras dificuldades que interferem nos indicadores dessa violência ${ }^{35}$.

No que concerne à coocorrência com outros tipos de violência, estudiosos apontam que a agressão sexual de crianças e adolescentes não representa um evento isolado, em geral, costuma estar acompanhada de outras situações violentas, como negligência, violência física, psicológica, entre outras ${ }^{36}$. Entretanto, os baixos índices de registros dos eventos concomitantes impossibilita a identificação dos casos e subestima a importância do reconhecimento dos mesmos. Pesquisas indicam que, em cada cinco casos, cerca de três sofrem outros episódios violentos, ou seja, $58 \%$ sofrem agressões diversas ${ }^{27}$. Esses dados sugerem que essas vítimas são muito susceptíveis aos problemas clínicos, psicossociais e comportamentais decorrentes do trauma, na dependência das características do evento, assim como do suporte familiar e social ${ }^{17,27}$. Os resultados do presente estudo que mostraram maiores coeficientes de outras violências concomitantes à violência sexual, mais expressivos a partir de 2007 (entre 2007 a 2010), concordam com dados da literatura, em nível mundial, que apontam que a coocorrência mais frequentes é a violência física e psicológica $^{6,37}$.

Em Feira de Santana, os achados deste estudo mostraram que mais de $20 \%$ dos casos de agressão sexual estavam relacionados à negligência, violência física e psicológica, cuja incidência apresentou aumento considerável, a partir da segunda metade da década. Esses resultados corroboram com a literatura nesta área, realizadas em distintas regiões do Brasil e outros paí$\operatorname{ses}^{13,14,34,36,38}$. Estudiosos têm mostrado que o abuso na infância e adolescência, assim como outras experiências adversas estão inter-relacionados e não ocorrem de forma independente. Importa ressaltar o consenso da literatura quanto à variação das taxas de violência sexual nos diversos contextos, em decorrência de fatores relacionados ao fenômeno, como a revelação pela vítima, a identificação, a denúncia e a notificação pela família, amigos, comunidade, assim como ao processo da investigação pelas instâncias de referência e posterior registro dos casos. Da mesma forma, esses indicadores sofrem influência de fatores relacionados ao processo de pesquisa (população e tipo de estudo, método e objetivos $)^{14,34}$. Portanto, vale assinalar a importância de estudos que investigam as múltiplas formas de vitimização, uma vez que os indicadores relacionados às manifestações individuais podem subestimar outras formas de exposição de crianças e adolescentes, assim como os respectivos fatores de risco associados ${ }^{19,27}$.

Nos EUA, estudos multicêntricos, confirmam essas evidências: Em 2009, pesquisa sobre a concomitância de episódios violentos, com amostra nacional de 4.053 indivíduos na faixa etária de 2 a 17 anos evidenciou que $66 \%$ dos participantes experimentaram múltiplas formas de vitimização, sendo que, destes, 30\% foram expostos a cinco ou mais tipos e 10\% experimentaram 11 ou mais manifestações ${ }^{18}$; em 2012, estudo com 2.017 crianças de 2-9 anos, observou exposição a diversos tipos de violência na família (tais como abuso físico ou sexual, maus-tratos emocionais, negligência infantil, vitimização por irmão, e testemunho de violência familiar), na maioria das vezes, relacionadas a relações familiares disfuncionais, tais como abuso de álcool e outras drogas, prisão, desemprego e desagregação fami$\operatorname{liar}^{19}$. Pesquisa realizada em San-Diego (EUA), com 8.629 adultos, constatou que dois terços dos participantes $(67,3 \%)$ foram expostos na infância, pelo menos, a uma categoria de abuso e a outras experiências adversas: 86,5\% relataram terem sido expostos a uma experiência adicional; $52 \%$ a três episódios e $38,5 \%$ quatro ou mais exposições $^{38}$.

No Canadá, foi verificada, entre crianças e adolescentes, a coocorrência das violências sexual, física e psicológica: 19\% dos rapazes e 26\% das moças ${ }^{12}$. 
Os achados da presente pesquisa, especialmente no que se refere às proporções da violência física e psicológica, concordam com estudos realizados em outras regiões do país, que identificaram mais de um tipo de violência concomitante aos casos de agressão sexual ${ }^{13,14,30}$. Estudo realizado em São Paulo, com 4.085 registros de casos de violação sexual na faixa até 15 anos, observou que a ocorrência isolada correspondeu a $33,2 \% ; 7,1 \%$ tiveram concomitantemente violência física e 3,0\% violência psicológica ${ }^{36}$.

A importância da identificação dos casos de episódios violentos em concomitância à agressão sexual possibilita conclusões precisas sobre o problema, o conhecimento da real magnitude do fenômeno, bem como o efeito cumulativo dessa adversidade e o comprometimento gerado às vítimas, podendo explicar a alta vulnerabilidade pelas perigosas e graves formas de vitimização 9,20,36. Pesquisadores sugerem que as vítimas submetidas a várias vitimizações encontram-se nas faixas etárias mais elevadas, em função das exposições acumuladas com a idade, sendo muito frequente na adolescência e no sexo masculino?.

No presente estudo observou-se baixa proporção de registro da violência psicológica, no mesmo evento da violação sexual, cuja principal consequência é o estresse pós-traumático ${ }^{6}$. Esses baixos índices, possivelmente, são decorrentes de sub-registro. A notificação adequada das violências perpetradas no mesmo evento está diretamente relacionada à eficiência profissional, cuja formação requer conhecimentos específicos para identificação e notificação dos múltiplos aspectos relacionados ao caso, os quais constituem importantes ferramentas para o adequado encaminhamento.

Outro achado importante da presente pesquisa foi que, em se tratando da ocorrência de violência sexual concomitante com a física, na maioria dos casos o abusador era desconhecido da vítima, concordando com dados da pesquisa de $\mathrm{Cruz}^{39}$ que indica como mais frequente esse tipo de associação, pela possível rejeição e falta de colaboração com o processo de violação, onde o perpetrador agride fisicamente para conter ou intimidar $6,37,39$. Esse tipo de abusador (oportunista ou pedófilo) é ocasional, raramente comete abusos com a mesma vítima, procurando novas para não ser reconhecido e denunciado, dificultando assim o processo de identificação e responsabilização ${ }^{39}$.

No estudo de Feira de Santana, as proporções de estupro e incesto foram semelhantes, com variações ao longo do período, cujas ocorrências foram originadas no contexto domiciliar, ou ambiente social da vítima, concordando com pesquisas que relatam resultados semelhantes ${ }^{33}$. Muitos estudos destacam o domicílio da vítima ou do agressor, assim como algum local da comunidade, como ambientes privilegiados para a prática da violação sexual perpetrada em crianças e adolescentes, trazendo a reflexão de que, em algumas situações o ambiente familiar e social pode não constituir segurança e proteção. A omissão, a ausência de testemunhas e a cumplicidade familiar ou de terceiros (amigos, vizinhos), quanto à denúncia, constituem importantes agravantes do evento e consequências, somadas às dificuldades inerentes ao processo de revelação pela vítima ${ }^{31,35}$. Nos EUA, levantamento nacional abordando crianças e adolescentes de 0 a 17 anos que foram agredidas sexualmente revelou que $14 \%$ ocorreram no ambiente domiciliar da víti$\mathrm{ma}^{16}$. Quanto ao local de ocorrência no ambiente extrafamiliar, os dados do presente estudo apontaram resultados que corroboram com outras pesquisas em nível nacional e internacional ${ }^{16}$. Vale salientar que a principal categoria de agressor observada refere-se a pessoas conhecidas da vítima, tanto no ambiente familiar, como extrafamiliar concordando com achados desses estudos ${ }^{16}$.

No presente estudo, o Sistema "Disque 100" destacou-se como principal veículo de captação das denúncias, concordando com pesquisas que apontam o anonimato como importante estratégia de viabilização da sindicância e identificação dos casos, pelas instâncias de referência, como os CREAS. O anonimato garante o não envolvimento com o caso e preserva a identidade do denunciante; por outro lado, a falta de detalhamento das informações e o receio na revelação da própria identidade podem dificultar a obtenção de dados que contribuem para a confirmação e elucidação do caso $^{40}$. Estas possibilidades orientam quanto à necessidade de sensibilizar a população sobre as características do sigilo, asseguradas pelo sistema de registro, visto a importância dos detalhes das informações, para os possíveis encaminhamentos e resolutividade dos casos.

Apesar da mãe da vítima ter sido também identificada como importante fonte de denúncia nesse e em outros estudos, essa frequência ainda é considerada baixa. Múltiplos fatores podem interferir para a omissão materna, como a tentativa em manter a representação social da família, evitando desenlaces. Denunciar o incesto pelo pai ou companheiro representa reconhecer o pró- 
prio fracasso no papel de esposa, assim como de mãe, importante elemento protetor e formador da família. Diferentes motivos levam a genitora e outros membros da família, amigos e vizinhos a omitir e negar a violação, contribuindo para o baixo impacto de denúncias e subnotificação de $\operatorname{casos}^{32}$. A revelação pela própria vítima é um processo complicado, pelos diferentes aspectos, valendo destacar que nesta etapa os indivíduos encontram-se em franco processo de desenvolvimento psicossocial. Crianças e adolescentes podem ficar submetidos ao poder do agressor por longos períodos, de acordo com o tipo de vinculação e credibilidade das pessoas de referência, fatores estes que têm impacto decisivo na gravidade do acometimento psicossocial e comportamental das vítimas ${ }^{16,25}$. Na atual pesquisa, os outros setores, como saúde e educação mostraram baixas proporções de denúncias mesmo vinculadas à obrigatoriedade e à aplicação de multas em casos de omissão da notificação.

Para concluir, vale assinalar as limitações do presente estudo que lida com dados secundários, os quais podem apresentar subnotificação e ausência de algumas variáveis importantes, ao longo do decênio. Assinala-se que os resultados apresentados dizem respeito aos registros de casos denunciados nas instâncias de referência, não retratando a real incidência deste fenômeno no município, considerando o repertório de dificuldades, mundialmente conhecidas, para a identificação e a notificação de cada caso de violação sexual perpetrada em crianças e adolescentes.

\section{Considerações finais}

Os resultados deste estudo apontam para a importância de investigações abordando a coocorrência de outras violências à sexual, uma vez que possibilitam melhor compreensão e avaliação mais precisa do impacto deste fenômeno para as vítimas, identificação de um maior número de crianças e adolescentes em situação de risco e intervenções mais abrangentes.

Estudos que envolvem levantamento de indicadores contribuem para dar visibilidade ao fenômeno. A utilização dos sistemas oficiais de registros permite conhecer taxas e tendências que indicam a magnitude e as características do problema, orientando quanto às possíveis medidas de intervenção e controle. Pesquisar o perfil e os coeficientes de incidência da violência sexual e outras violências concomitantes perpetradas em crianças e adolescentes, ao longo de uma década pode contribuir para subsidiar políticas e práticas efetivas pelos diversos setores, assim como divulgar o conhecimento científico nessa área.

Destaca-se a importância da formação de "Redes Institucionais" que integrem serviços já existentes, cujas políticas viabilizem recursos direcionados à formação profissional, na identificação, notificação e encaminhamentos, frente aos casos, proporcionando assim a implementação de estratégias eficazes de interrupção do ciclo de vitimização.

\section{Colaboradores}

JR Oliveira, MCO Costa, MTR Amaral, CA Santos, SG Assis e OC Nascimento participaram igualmente de todas as etapas de elaboração do artigo.

\section{Agradecimentos}

À Fundação de Amparo à Pesquisa do Estado da Bahia/FAPESB pelo apoio à presente pesquisa. 


\section{Referências}

1. Krug ED, Dalberg LL, Mercy JA, Zwi AB, Lozano R. Relatório Mundial sobre Violência e Saúde. Genebra: Organização Mundial da Saúde (OMS); 2002.

2. Minayo MCS. Violência: um velho-novo desafio para a atenção à Saúde. Rev Bras Educ Med 2005; 29(1):55-63.

3. Motti AJA, Contini MLJ, Amorim SMF, organizadores. Consolidando a Experiência do PAIR. Campo Grande: Editora UFMS; 2008.

4. Ferreira AL, Souza ER. Análise de indicadores de avaliação do atendimento a crianças e adolescentes em situação de violência. Cad Saude Publica 2008; 24(1):28-38.

5. Bernier MJ, Hébert M, Vezina D. Symptômes de dissociation chez les enfants d'âge préscolaire ayant dévoilé une aggression sexuelle. Journal Internacional de Victimologie 2011; 9(2):318-332.

6. Simoneau AC, Hébert M, Tourigny M. Recension des études évaluatives des interventionsde groupe destinées aux enfants de six à douze ans victimes d'agression sexuelle. Rev Psychoeduc 2008; 37(2): 245-288.

7. Berthelot N, Langevin R, Hébert M. L'association entre la victimisation sexuelle dans I'enfance de la mère et les troubles de comportemente chez I'enfant victim d'agression sexuelle. Journal International de Victimologie 2012; 10(1):8-20.

8. Hébert M, Tourigny M, Cyr M, McDuff PMS, Joly J. Prevalence of Childhood Sexual Abuse and Timing of Disclosure in a Representative Sample of Adults From Quebec. Can J Psychiatry 2009; 54(9): 631-636.

9. Turner HA, Finkelhor D, Ormrod R. Poly-victimization in a National Sample of Children and Youth. Am J Prev Med 2010; 38(3):323-330.

10. Finkelhor D, Ormrod RK, Chaffin M. Juveniles Who Commit Sex Offenses Against Minors. Washington: Office of Juvenile Justice and Delinquency Prevention.; 2009.

11. Sabina C, Straus MA. Poly-victimization by Dating Partners and Mental Health Among U.S. College Students. Violence Vict 2008; 37(23):667-682.

12. Sears HA, Byers SE, Price LE. The co-occurrence of adolescent boys' and girls' use of psychologically, physically, and sexually abusive behaviors in their dating relationships. J Adolesc. 2007; 30(38):487-504.

13. Hamby S, Finkelhor D, Turner H. Teen Dating Violence: Co-Occurrence With Other Victimizations in the National Survey of Children's Exposure to Violence (NatSCEV). Psychol Violence 2012; 2(2): 111-124.

14. Barreira AK, Lima MLC, Avanci JQ. Coocorrência de violência física e psicológica entre adolescentes namorados do Recife, Brasil; Prevalência e fatores associados. Cien Saude Colet 2013; 18(1):233-243.

15. International Society for Preventions of Child Abuse and Neglect (ISPCAN). World Perspectives on Child Abuse. $7^{\text {th }}$ Edition. Aurora: ISPCAN; 2006.

16. Finkelhor D, Hammer H, Sedlar AJ. Sexually Assaulted Children: National Estimates and Characteristics. Philadelphia: National Incidence Studies of Missing, Abductes, Runaway, and Thrownaway Children; 2008.
17. Trocmé NM, Fallon B, Maclaurin B, Daciuk J, Felstiner C, Black T. Major findings from the Canadian incidence study of reported child abuse and negect. Child Abuse Negl 2003; 27(12):1427-1439.

18. Finkelhor D, Ormrod R, Turner HA, Holt M. Pathways to poly-victmization. Child Maltreat 2009; 14(4):316-329.

19. Turner HA, Finkelhor D, Ormrod R, Hamby S. Family context, victimization, and child trauma symptoms: variation in Safe, stable, and nurturing relationships during early and middle childhood. Am J Orthopsychiatry 2012; 82(2):209-219.

20. Finkelhor D, Ormrod RK, Turner HA. Lifetime assessment of poly-victimization in a national sample of children e youth. Child Abuse Negl 2009; 33(7):403-411.

21. Ford JD, Elhai JD, Connor DF, Frueh BC. Polyvictimization and Risk of Posttraumatic, Depressive, and Substance Use Disorders and Involvement in Delinquency in a National Sample of Adolescents. J Adolesc Health 2010; 46(6):545-552.

22. Mascarenhas MDM, Malta DC, Silva MMA, Lima CM, Carvalho MGO, Oliveira VLA. Violência contra a criança: revelando o perfil dos atendimentos em serviços de emergência, Brasil, 2006 e 2007. Cad Saude Publica 2010; 26(2):347-357.

23. Costa MCO, Carvalho RC, Santana MAO, Silva IMS, Silva MR. Avaliação do Programa Nacional de Ações Integradas e Referenciais (PAIR) para o enfrentamento da violência sexual contra crianças e adolescentes, em Feira de Santana, Bahia. Cien Saude Colet 2010; 15(2):563-574.

24. Brasil. Lei n. ${ }^{\circ} 8.069$, de 13 de julho de 1990. Dispõe sobre a proteção integral à criança e ao adolescente. Diário Oficial da União 1990; 13 jul.

25. Scherer EA, Scherer ZAP. A criança maltratada: uma revisão da literatura. Rev Lat Am Enfermagem 2000; $8(4): 22-29$.

26. Willians ICA, Padovani RC, Araújo EAC, Pereira ACS, Ormenõ GR, Eisenstein E. Fortalecendo a rede de proteção da criança e do adolescente. São Carlos: Pedro e João Editores; 2009.

27. Finkelhor D, Turner H, Hamby S, Ormrod R. Polyvictimization: children's exposure to multiple types of violence, crime, and abuse. National Survey of Children's Exposure to Violence. Juvenile Justice Bulletin 2011; Oct:1-12.

28. Silva CF, Leite AJM, Almeida NMGS. Linkage entre bancos de dados de nascidos vivos e óbitos infantis em município do Nordeste do Brasil: qualidade dos sistemas de informação. Cad Saude Publica 2009; 25(7):1552-1558.

29. Brasil. Ministério da Saúde (MS). Conselho Nacional de Saúde. Resolução no 196 de 10 de outubro de 1996. Diretrizes e Normas Regulamentadoras de Pesquisas Envolvendo Seres Humanos. Diário Oficial da União 1996; 16 out.

30. Arcos E, Uarac M, Molina I. Impacto de la violência doméstica en la salud infantil. Rev Med Chil 2003; 131(12):1454-1462. 
31. Ribeiro MA, Ferriani MGC, Reis JN. Violência sexual contra crianças e adolescentes: características relativas à vitimização nas relações familiares. Cad Saude Publica 2004; 20(2):456-464.

32. Pfeiffer L, Salvagni EP. Visão atual do abuso sexual na infância e adolescência. J Pediatr (Rio J) 2005; 81(Supl. 5):S197-S204.

33. Habigzang LF, Koller SH, Azevedo GA, Machado PX. Abuso sexual e dinâmica familiar: aspectos observados em processos jurídicos. Psicologia: Teoria e Pesquisa 2005; 21(3):341-348.

34. Martins CBG, Mello-Jorge MHP. A violência contra crianças e adolescentes: características epidemiológicas dos casos notificados aos Conselhos Tutelares e programas de atendimento em município do Sul do Brasil, 2002 e 2006. Epidemiologia em Serviços de Saúde 2009; 18(4):315-334.

35. Hohendorff JV, Habigzang LF, Koller SH. Violência sexual contra meninos: dados epidemiológicos, características e consequências. Psicologia USP 2012; 23(2):395-415.

36. Finkelhor D, Ormrod RK, Turner HA. Polyvictimization: a neglected component inchild victimization Child Abuse Negl 2007; 31(1):7-26.

37. Gawryszewski VP, Valencich DMO, Carnevalle CV, Marcopito LF. Maus-tratos contra criança e ao adolescente no Estado de São Paulo, 2009. Rev Assoc Med Bras 2012; 58(6):659-665.

38. Dong M, Anda RF, Felitti VJ, Dube SR, Williamson DF, Thompson TJ, Loo CM, Giles WH. The interrelatedness of multiple forms of childhood abuse, neglect, and household dysfunction. Child Abuse Negl 2004; 28(7):771-784.

39. Cruz RA. Abuso Sexual na Infância: Conceito Básicos e Medidas de Prevenção. In: Silva AS, Senna E, Kassar MCM, organizadores. Exploração Sexual de Crianças e Adolescentes. Brasília: Organização Mundial do Trabalho (OMT); 2005. p. 71-88.

40. Di Lorenzi DRS, Pontalti L, Flech RM. Maus tratos na infância e adolescência: análise de 100 casos. Revista Científica da AMECS 2001; 10(1):47-52.

Artigo apresentado em 30/09/2013

Aprovado em 18/10/2013

Versão final apresentada em 24/10/2013 